Research Article

\title{
Antipsychotic medication prescribing trends in a tertiary care hospital
}

\author{
Riyaz Ahmed Siddiqui*, Tanaji Ramaji Shende, Harshal Murlidhar Mahajan, \\ Archana Borkar
}

Department of Pharmacology,

NKPSIMS, Nagpur,

Maharashtra, India

Received: 03 May 2016

Accepted: 03 June 2016

*Correspondence to:

Dr. Riyaz Ahmed Siddiqui,

Email: riyaz_ammar@

rediffmail.com

Copyright: (C) the author(s), publisher and licensee Medip Academy. This is an openaccess article distributed under the terms of the Creative Commons Attribution NonCommercial License, which permits unrestricted noncommercial use, distribution, and reproduction in any medium, provided the original work is properly cited.

\begin{abstract}
Background: In psychiatric practice, antipsychotic drugs are widely used group of drugs. Numbers of drugs are available in this group with diverse type of mechanisms and efficacy/safety profile. The objective for the study was to identify the prescription pattern of antipsychotic drugs in the centre for determination of current trends.

Methods: The retrospective study was conducted at NKP Salve Institute of Medical Sciences and Research Centre, Nagpur, Hospital, Maharashtra, India case record files of the department of psychiatry for the year 2014-2015 were analysed for a total of 300 cases. Antipsychotic drugs received by the patient for psychiatric disorders like schizophrenia, mood disorders (major depression or mania) with psychotic features, substance induced psychosis, dementia and delirium with psychotic features, brief psychotic disorder, delusional disorder, schizoaffective disorders etc. were noted and analysed for prescription pattern of antipsychotic drugs.

Results: Out of 300 cases that were analysed, olanzapine was the most commonly used antipsychotic drug i.e. in $51 \%$ of patients followed by resperidone in $23 \%$ and quetiapine in $13 \%$ of patients. Other drugs used were aripiprazole in $6 \%$, clozapine in in $5.3 \%$, iloperidine in $5 \%$, trifluperazine in $2 \%$ and triflupromazine in $1 \%$ of patients. Injectable haloperidol and the anticholinergic promethazine were used as on required basis.

Conclusions: Atypical antipsychotics are more commonly used as compared to the typical ones. Atypical antipsychotics like olanzapine, resperidone and quetiapine are preferred because of their lesser propensity to cause extrapyramidal adverse effects and they also helps in improving negative symptoms of schizophrenia.
\end{abstract}

Keywords: Atypical antipsychotic, Extrapyramidal adverse effect, Neuroleptics

\section{INTRODUCTION}

Antipsychotics are a class of agents which are able to reduce psychotic symptoms in a wide range of conditions like schizophrenia, bipolar disorder, psychotic depression, senile psychosis, various organic psychosis and drug induced psychosis. Antipsychotic prescription patterns are fundamentally different across countries and even regions due to variations in factors including health care policies, availability and cost of drugs, psychiatric training and preferred treatment modalities. Psychosis is a symptom of mental illnesses characterized by a distorted or non-existent sense of reality. Psychotic disorders have different etiologies, each of which demands a unique treatment approach. ${ }^{1}$ Currently a large number of atypical antipsychotics available in the market are endorsed as better option for treating schizophrenia than the typical antipsychotics. Medical aspects focus on the need to balance between the risk and the benefits. The benefits are assessed on the basis of drug efficacy in preventing, relieving and curing diseases or their symptoms and complications. Potential risks include short term and long term adverse effects. ${ }^{2}$

Application of pharmacoepidemiological studies often reveals the large differences that exist between the conditions of premarketing trials (done on a limited sample population) and those of actual practice several years into the market life of a pharmaceutical product. Clinical trial data must therefore be complemented by measurement of any eventual distortions and estimation of the real-life effectiveness and safety of the medicines. ${ }^{3}$ 
Data from different parts of the world indicate increasing prescriptions for drugs used for mental disorders, particularly antidepressants and antipsychotics. Studies of antipsychotic prescribing show increases over the past two decades in a variety of countries. ${ }^{4}$ The rapidly expanding field of psychopharmacology is challenging the traditional concepts of psychiatric treatment and research, and is constantly seeking new and improved drugs to treat psychiatric disorders. In this way, psychiatrists are continuously exposed to newly introduced drugs that are claimed to be safe and more efficacious. Although psychotropic medications have had a remarkable impact on psychiatric practice that legitimately can be called revolutionary, their utilization and consequences on real life effectiveness and safety in actual clinical practice need continuous study. ${ }^{5}$

Availability of wide variety of antipsychotic drugs and globally their different pattern of prescription indicates that different regional population need different antipsychotic drug. Therefore this study was undertaken to analyse the prescription pattern of various antipsychotic drugs in this part of central India hospital so that the current trend can be identified which will be useful for the management of psychiatric disorders.

\section{METHODS}

It is a retrospective record based study that is carried out at NKP salve institute of medical sciences and RC, Nagpur. Ethical committee permission was obtained prior to the study. Hospital case record files of the department of psychiatry for the year 2014-2015 were analysed for a total of 300 cases. The various antipsychotic drugs received by the patient like typical antipsychotic and atypical antipsychotic for various psychiatric disorders like schizophrenia, mood disorders (major depression or mania) with psychotic features, substance induced psychosis, dementia and delirium with psychotic features, brief psychotic disorder, delusional disorder, schizoaffective disorders etc. were noted. Data was recorded in case record from and analysed using Microsoft excel program.

\section{RESULTS}

We have analysed a total number of 300 hospital case record files of the department of psychiatry of the year 2014-2015. The various antipsychotic drugs prescribed to the patients like typical antipsychotic and atypical antipsychotic for various psychiatric disorders like schizophrenia, mood disorders (major depression or mania) with psychotic features, substance induced psychosis, dementia and delirium with psychotic features, brief psychotic disorder, and delusional disorder, schizoaffective disorders etc. were noted. Olanzapine was the was the most commonly used antipsychotic drug i.e. in $51 \%$ of patients followed by resperidone in $23 \%$ and quetiapine in $13 \%$ of patients. Other drugs used were aripiprazole in $6 \%$, clozapine in in $5.3 \%$, Iloperidine in
$5 \%$, trifluperazine in $2 \%$ and triflupromazine in $1 \%$ of patients. Injectable haloperidol and the anticholinergic promethazine were used as on required basis.

Table 1: Depicting drug received by the patient and their percentage.

\begin{tabular}{|lll|}
\hline Drug received & No. of patients & $\%$ of patients \\
\hline Olanzapine & 153 & 51 \\
\hline Resperidone & 69 & 23 \\
\hline Quetiapine & 39 & 13 \\
\hline Aripiprazole & 18 & 6 \\
\hline Clozapine & 16 & 5.3 \\
\hline Ilobril & 15 & 5 \\
\hline Trifluperazine & 6 & 2 \\
\hline Triflupromazine & 3 & 1 \\
\hline
\end{tabular}

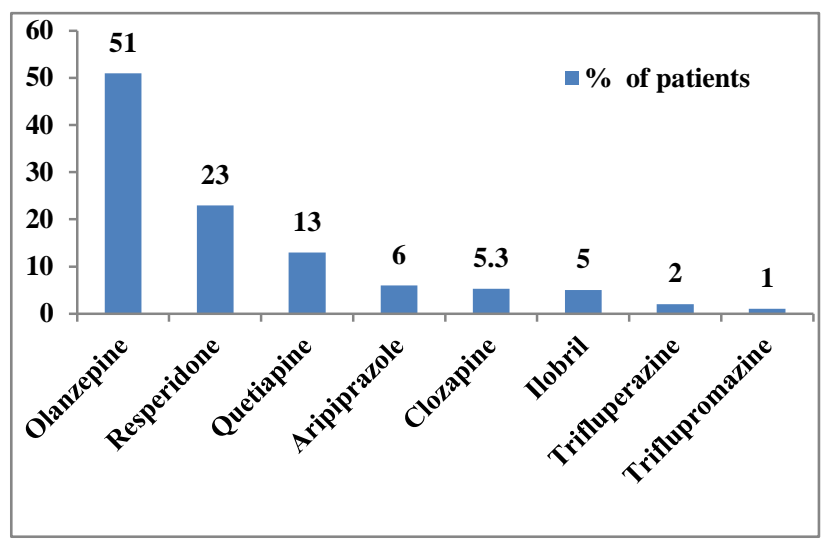

Figure 1: Depicting drug received by the patient and their percentage.

\section{DISCUSSION}

In the present study olanzapine was the most commonly used antipsychotic drug i.e. in $51 \%$ of patients followed by resperidone in $23 \%$ and quetiapine in $13 \%$ of patients. Other less commonly used drugs were aripiprazole in $6 \%$, clozapine in in $5.3 \%$, iloperidine in $5 \%$, trifluperazine in $2 \%$ and triflupromazine in $1 \%$ of patients. Centorrino et al evaluated medication use in all McLean Hospital in patients treated with antipsychotic drugs during 3 months in 1998, the ranking of antipsychotic use for the 349 patients was olanzapine in $36 \%$, risperidone $17 \%$, quetiapine $17 \%$, perphenazine $10 \%$, clozapine $6 \%$, and haloperidolin $6 \%$; other typical neuroleptics were used for $3 \%$ or less of the patients . ${ }^{6}$ Banerjee et al in their study at a teaching hospital in schizophrenic patients found that olanzapine was the commonest antipsychotic drug prescribed in $34.3 \%$, followed by haloperidol in $28.6 \%$, combination of haloperidol and olanzapine $12.4 \%$, risperidone $17.4 \%$, clozapine $3.8 \%$, quetiapine $2.4 \%$ and aripiprazole $1 \%$ (atypical antipsychotics ) respectively. ${ }^{2}$ Blin et al carried out cross sectional survey by a random sample of 61 public and 112 private psychiatrists who registered for one month all schizophrenic adult outpatients treated with a neuroleptic drug. Psychiatrists 
included 934 patients in the public care setting and 927 patients in the private care setting. An atypical neuroleptic was prescribed for $63.0 \%$ of patients and a classic neuroleptic for $49.7 \%$, an association of neuroleptics for $22.0 \%$ and in both settings, the most prescribed neuroleptics were olanzapine $(28.0 \%)$ and risperidone $(18.6 \%){ }^{7}$ Also Deshmukh et al in a prospective, cross sectional and observational study over 22 weeks on 515 newly diagnosed psychiatric out patients observed that apart from antidepressants $(60.4 \%)$, in antipsychotics category, olanzapine use was highest $(26.6 \%)$. Piparva et al in a prospective cross sectional study of 6 months duration in outdoor patients (OPD) of the psychiatry department found that atypical antipsychotic drugs $(43.83 \%)$ were prescribed more frequently than the typical antipsychotic drugs $(26.32 \%) .^{5}$ Ghosh et al in a cross-sectional, unicentric study in the psychiatry outpatient department (OPD), of a tertiary care, medical college and hospital found that atypical antipsychotics are prescribed more commonly than typical antipsychotic and among the atypical antipsychotics olanzapine is most commonly prescribed. ${ }^{9}$ Dutta et al in their study on psychotropic drug use among depression patients attending private psychiatric practitioners found that SSRIs $(39.17 \%)$ were the most commonly prescribed class of antidepressants and among patients, $18.48 \%$ antipsychotics were prescribed to patients; olanzapine and risperidone were the most commonly prescribed. Trihexyphenidyl was the most common anticholinergic prescribed. ${ }^{10}$ Grohmann et al in their study analyse data on drug use in 1995 and 2001 assessed at two reference days per year and per hospital within the drug safety program AMSP were compared for 10 hospitals found that atypical neuroleptics (NL) were used in $59.9 \%$ of patients on NLs in 2001 (16.7\% in 1995), most frequently olanzapine, risperidone, clozapine, and quetiapine, in this order. $39 \%$ of patients still received typical NLs in antipsychotic indication $(77.1 \%$ in 1995$)$, and $30.8 \%$ received typical hypnosedative NLs (38.1\% in 1995). ${ }^{11}$ All the above findings suggests that atypical antipsychotics like olanzapine and respiridone are the commonly prescribed drugs as compared to the atypical ones which is in accordance with our study. Increase use of atypical antipsychotic agents like olanzapine, risperidone, quetiapine and ziprasidone can be because they have provided a better control of symptoms and reduced the chances of adverse effects especially the extrapyramidal ones in contrast with typical antipsychotic drugs and also proved to be better in improving negative symptoms, cognitive dysfunction and also efficacious in antipsychotic resistant cases. ${ }^{2}$

Doshi et al in their cross-sectional, prospective drug utilization study in outdoor patient of Psychiatry in a tertiary care hospital among 600 patients, found that most common disorder was depression $208(34.66 \%)$ followed by schizophrenia 192 (32.00\%), and the most common prescribed drugs are diazepam $74.55 \%$. Next in line are risperidone $59.76 \%$, olanzapine, $40.23 \%$, trihexyphenidyl $43.75 \%$, and clozapine $13.01 \% .^{12}$ Iliyaz et al in a retrospective cross sectional study conducted at Hyderabad, analysed 500 prescription of patients with neuropsychiatric disorder observed that resperidone was $40.8 \%$ prescribed, olanzapine $4.02 \%$, quetiapine $8.62 \%$, amisulpiride $4.02 \%$ and aripiprazole $0.57 \%$ prescribed. Also in anxiety disorder as a concomitant medicine resperidone was prescribed in $29.07 \%$ and olanzapine in $3.54 \% .^{13}$ In these above two studies though atypical antipsychotics were more commonly prescribed but respiridone was more commonly prescribed than olanzepine. Also Thakkar et al in a drug utilization study 600 prescriptions for different psychiatric disorders found that most commonly prescribed drugs for schizophrenia, bipolar disorders, depression and anxiety disorders were trifluoperazine + trihexiphenydyl $(63.9 \%)$, carbamazepine (17.2\%), amitriptyline (34.9\%), and diazepam (23.8\%), respectively. In this one study combination of typical antipsychotic (trifluperazine) with an anticholinergic (trihexiphenydyl) was used more commonly than atypical antipsychotic is not in accordance with our study findings. ${ }^{14}$

The FDA has approved the use of selected antipsychotic medications for children and adolescents for treatment of severe conduct problems that are resistant to other forms of treatment. Specific child psychiatric disorders include Tourette's syndrome and behavioural symptoms associated with autistic disorder, childhood schizophrenia, and bipolar disorder. ${ }^{15}$ National guidelines do recommend antipsychotics for the relatively rare condition of psychotic depression and as a possible intervention for treatment-resistant cases of severe depression and OCD. In clinical practice, these agents may be prescribed 'offlabel' for patients who do not have a diagnosis of SMI (severe mental illness) in their clinical notes. They are sometimes used to augment antidepressants in complex or treatment-resistant cases of OCD, anxiety and personality disorders. For borderline personality disorder short-term treatment with antipsychotics (up to a week) may be beneficial in crisis situations or when comorbid psychotic symptoms occur. ${ }^{16}$

\section{CONCLUSION}

Antipsychotics are one of the commonly used medications for schizophrenia and other psychiatric disorders. Of the antipsychotics, atypical antipsychotics like olanzapine, resperidone and quetiapine were most commonly used. Use of typical antipsychotics is declining. Atypical agents are preferred because of their better symptoms control and reduced chances of the extrapyramidal adverse effects and also in improving negative symptoms, cognitive dysfunction and also efficacious in antipsychotic resistant cases.

Funding: No funding sources Conflict of interest: None declared

Ethical approval: The study was approved by the Institutional Ethics Committee 


\section{REFERENCES}

1. Paul PK, Konwar M, Das S. To study the prescribing pattern of antipsychotic drugs in a tertiary care hospital of Assam. International Journal of Pharmacy and Pharmaceutical Sciences. 2014;6(4):435-7.

2. Banerjee I, Roy B, Sathian B, Banerjee I, Chakraborty PK, Saha. A socio demographic profile and utilization pattern of antipsychotic drugs among schizophrenic inpatients: a cross sectional study from western region of Nepal. BMC Psychiatry 2013;13:96.

3. Lahon K, Shetty HM, Paramel A, Sharma G. Pharmacoepidemiological study of antipsychotics in the psychiatry unit of a tertiary care hospital: A retrospective descriptive analysis. Int $\mathbf{J}$ Nutr Pharmacol Neurol Dis. 2012;2:135-41.

4. Ilyas S, Moncrieff J. Trends in prescriptions and costs of drugs for mental disorders in England, 19982010. The British Journal of Psychiatry.2012;200(5)393-8.

5. Piparva KG, Parmar DM, Singh AP, Gajera MV, Trivedi HR. Drug utilization study of psychotropic drugs in outdoor patients in a teaching hospital. Indian J Psychol Med. 2011;33(1):54-8.

6. Centorrino F, Eakin M, Bahk WM, Kelleher JP, Goren J, Salvatore P, et al. Inpatient antipsychotic drug use in 1998, 1993, and 1989. The American Journal of Psychiatry. 2002;159(11):1932-5.

7. Blin P, Olié JP, Sechter D, Petitjean F, Cialdella P, Gérard A, et al. Neuroleptic drug utilization among schizophrenic outpatients. Rev Epidemiol Sante Publique. 2005;53(6):601-13.

8. Deshmukh SA, Tteli SE. Evaluation of psychotropic drugs use pattern among outpatients attending psychiatry department at government medical college and hospital, Nagpur: a cross sectional study. Int $\mathbf{J}$ Pharm Bio Sci. 2012;3(3):428-36.
9. Ghosh S, Swati B, Dalai CK. Antipsychotic prescribing pattern in a tertiary care hospital of eastern India. Journal of Drug Delivery and Therapeutics. 2013;3(4):38-42.

10. Dutta S, Kaul V, Beg MA, Sindhu S, Singh NK, Bawa $S$, et al. A psychotropic drug use study among depression patients attending private psychiatric practitioners of Dehradun, Uttarakhand. Int J Med Sci Public Health. 2015;4(5):634-8.

11. Grohmann R, Engel RR, Geissler KH, Rüther E. Psychotropic drug use in psychiatric inpatients: recent trends and changes over time-data from the AMSP study. Pharmacopsychiatry. 2004;37(Suppl 1):S27-38.

12. Doshi CM, Hedamba R, Darji NH, Patel B , Trivedi HR, Tiwari D. Drug utilization study of psychotropic drugs in outdoor patients in a tertiary care hospital attached with a medical college. International Journal of Basic and Clinical Pharmacology. 2015;4(6).

13. Ilyaz MD, Baig MA, Rramakrishna, quadir MA, Fathima M, Abdul S. Drug utilization study of antipsychotics and its common adr's in the psychiatry opd of OHRC. International journal of Pharmacy and Pharmaceutical Sciences. 2014;6(9):162-5.

14. Thakkar KB, Jain MM, Billa G, Joshi A, Khobragade AA. A drug utilization study of psychotropic drugs prescribed in the psychiatry outpatient department of a tertiary care hospital. Journal of Clinical and Diagnostic Research. 2013;7(12):2759-64.

15. Harrison JN, Keller FC, Gross D. Antipsychotic medication prescribing trends in children and adolescents. J Pediatr Health Care. 2012;26(2):13945.

16. Marston L, Nazareth I, Walters K, David P, Osborn J. Prescribing of antipsychotics in UK primary care: a cohort study. BMJ Open. 2014;4:e006135.

Cite this article as: Siddiqui RA, Shende TR, Mahajan HM, Borkar A. Antipsychotic medication prescribing trends in a tertiary care hospital. Int $\mathbf{J}$ Basic Clin Pharmacol 2016;5:1417-20. 